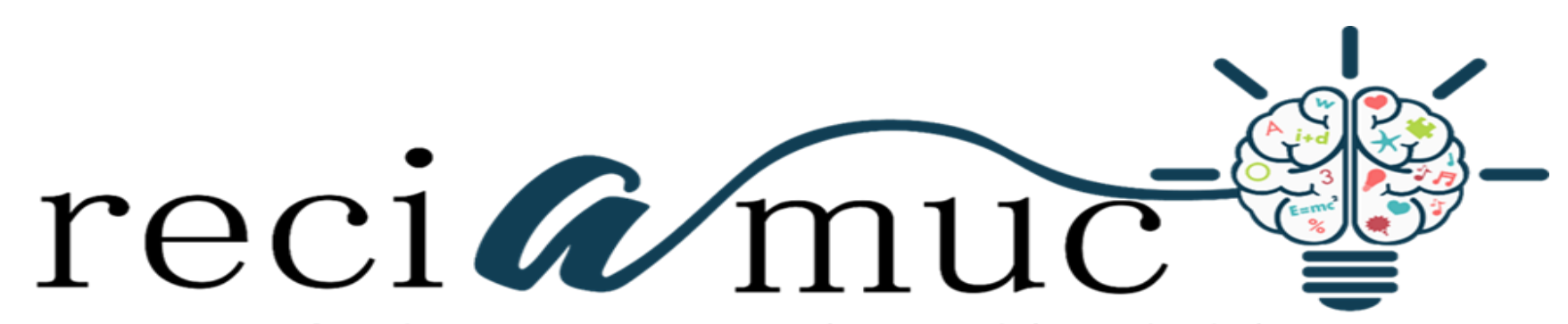

Revista cientifica de investigación actualización del mundo de las ciencias

\author{
Norma Betsabe Arellano Hernández ${ }^{\text {a; }}$ Karla Estefanía Burgos Cedeño ${ }^{\text {b; }}$ Karen \\ Mabel Sornoza Moreira ${ }^{c}$; Sandra Lissette Arregui Romero ${ }^{\mathrm{d}}$
}

Tratamiento de intoxicación aguda por acetaminofén

Acutaminofen acute intoxication treatment

Revista Científica de Investigación actualización del mundo de las Ciencias. Vol. 3

núm., 2, abril, ISSN: 2588-0748, 2018, pp. 488-502

DOI: $10.26820 /$ reciamuc/3.(2).abril.2019.488-502

URL: $\underline{\text { htp://reciamuc.com/index.php/RECIAMUC/article/view/350 }}$

Código UNESCO: 3205 Medicina Interna

Tipo de Investigación: Artículo de Revisión

(C) RECIAMUC; Editorial Saberes del Conocimiento, 2019

Recibido: 15/01/2019

Aceptado: 07/02/2019

Publicado: 01/04/2019

Correspondencia: betsita_123@hotmail.com
a. Médico; Investigador Independiente; Guayaquil, Ecuador; betsita_123@ hotmail.com
b. Médica Cirujana; Investigador Independiente; Guayaquil, Ecuador; karl1592@hotmail.com
c. Médico; Investigador Independiente; Guayaquil, Ecuador; kattita_sornoza@ @otmail.com
d. Médico; Investigador Independiente; Guayaquil, Ecuador; arreguilissette@ gmail.com 


\section{Tratamiento de intoxicación aguda por acetaminofén}

Vol. 3, núm. 2., (2019)

Norma Betsabe Arellano Hernández; Karla Estefanía Burgos Cedeño; Karen Mabel Sornoza Moreira; Sandra Lissette Arregui Romero

\section{RESUMEN}

El acetaminofén o paracetamol es el antitérmico y analgésico más usado en todo el mundo. Su venta libre lo hace percibir en la población como un fármaco inocuo, no obstante, está evidenciado que su sobredosificación trae efectos perjudiciales y daños al organismo. La intoxicación aguda con acetaminofén está asociada a riesgos hepáticos, coagulopatía, riesgos renales, compromiso neurológico y hasta la muerte. El objetivo fundamental de este estudio es plasmar el tratamiento de la intoxicación aguda con acetaminofén en la población infantil y adulta. El diseño de investigación que se llevó a cabo es de tipo documental o bibliográfico. El tratamiento básico de estas intoxicaciones es la administración de un antídoto llamado acetilcisteína, el cual tratará de eliminar el contenido del agente tóxico del organismo evitando, en la medida de lo posible, el daño hepático. En conclusión, no existe unificación de criterios a nivel internacional acerca de un régimen de administración del tratamiento con acetilcisteína, no obstante, se ha adoptado un método tradicional que ha ido variando en base a nuevos hallazgos y se ha modificado a regímenes más modernos que permiten tratar la intoxicación y evitar en mayor medida los efectos adversos del antídoto.

Palabras Claves: Tratamiento; Intoxicación; Aguda; Acetaminofén; Antídoto. 


\title{
Tratamiento de intoxicación aguda por acetaminofén
}

Vol. 3, núm. 2., (2019)

Norma Betsabe Arellano Hernández; Karla Estefanía Burgos Cedeño; Karen Mabel Sornoza Moreira; Sandra Lissette Arregui Romero

\begin{abstract}
Acetaminophen or acetaminophen is the most used antipyretic and analgesic in the world. Its free sale makes it perceived in the population as an innocuous drug, however, it is evident that its overdose brings harmful effects and damages to the organism. Acute poisoning with acetaminophen is associated with liver risks, coagulopathy, kidney risks, neurological compromise and even death. The main objective of this study is to capture the treatment of acute poisoning with acetaminophen in children and adults. The research design that was carried out is documentary or bibliographic. The basic treatment of these poisonings is the administration of an antidote called acetylcysteine, which will try to eliminate the content of the toxic agent of the organism avoiding, as far as possible, liver damage. In conclusion, there is no unification of criteria at international level about a regimen of administration of acetylcysteine treatment, however, a traditional method has been adopted that has varied based on new findings and has been modified to more modern regimes that allow treat poisoning and further avoid the adverse effects of the antidote.
\end{abstract}

Key words: Treatment; Poisoning; Acute; Acetaminophen; Antidote. 


\section{Tratamiento de intoxicación aguda por acetaminofén}

Vol. 3, núm. 2., (2019)

Norma Betsabe Arellano Hernández; Karla Estefanía Burgos Cedeño; Karen Mabel Sornoza Moreira; Sandra Lissette Arregui Romero

\section{Introducción.}

Uno de los problemas importantes de salud pública mundial está constituido por las intoxicaciones. Para el año 2004 un aproximado de 346.000 personas fallecieron en todo el mundo como consecuencia de una intoxicación no intencional, de estas muertes el $91 \%$ fueron producidas en países de bajo y mediano ingreso. (Organización Mundial de la Salud, 2017)

La intoxicación es capaz de lesionar o incluso matar a la persona que la sufre y puede surgir como consecuencia de tragar, tocar, inhalar o inyectar medicamentos, venenos, gases o productos químicos. Algunas sustancias son más peligrosas que otras, tal es el caso de los medicamentos, cuando se excede su dosis o concentración. Dentro de la población más sensible a intoxicaciones se encuentran los niños. (Mayo Clinic, 2019)

En el caso de ser un medicamento quien ocasiona el cuadro tóxico, nos encontramos en presencia de una intoxicación medicamentosa, las cuales son muy frecuentes, ya sean intencionales o no. Asimismo, existen niveles de intoxicación, dentro de las cuales la más común corresponde a la intoxicación aguda.

Para De la Torre (2014) la intoxicación aguda consiste en cualquier tipo de exposición, por ingesta, tacto o inhalación, a una o varias sustancias capaces de ocasionar lesiones en el organismo. (p. 281)

Por otra parte, Oliva \& Canals (2018) la definen como un "síndrome clínico secundario a la introducción brusca de un tóxico en el organismo, tanto de forma intencionada como accidental, lo que produce efectos nocivos en el individuo". (p. 52) 


\section{Tratamiento de intoxicación aguda por acetaminofén}

Vol. 3, núm. 2., (2019)

Norma Betsabe Arellano Hernández; Karla Estefanía Burgos Cedeño; Karen Mabel Sornoza Moreira; Sandra Lissette Arregui Romero

En este orden de ideas, este tipo de intoxicación puede llegar a lesionar el organismo de múltiples formas. Ante su presencia, la administración de antídotos es el tratamiento fundamental, que en muchos casos puede llegar a ser la única terapia vital e insustituible.

Dentro de los medicamentos causantes de más intoxicaciones cada año en todo el mundo se encuentra el acetaminofén o paracetamol.

Triviño, Martínez, \& Luaces, (2012) coinciden al respecto y señala que el "Grupo de Trabajo de Intoxicaciones de la Sociedad Española de Urgencias Pediátricas (SEUP) lo sitúan como la primera causa de intoxicación farmacológica en menores de 5 años”. Según encuestas de dicho grupo de trabajo, la ingesta accidental de paracetamol correspondió al 12,3\% del total de intoxicaciones y que el $22 \%$ de las intoxicaciones medicamentosas, estos datos obtenidos de un estudio realizado en 37 servicios de emergencias pediátricas durante el periodo octubre del año 2008 y septiembre del 2009. (p. 119)

En la actualidad, el acetaminofén o paracetamol es el analgésico-antipirético de mayor uso a nivel mundial y de libre comercialización. No necesita una prescripción médica, lo que hace que la mayoría de la gente subestimen su toxicidad. Esto conlleva a que el acetaminofén sea el causante número uno de intoxicaciones medicamentosas, ya sea por desconocimiento de su dosificación o por sobredosis voluntaria.

La concepción de este medicamento de venta libre, como la de otros de este tipo, es que son inocuos, muchas veces administrado sin supervisión médica y sobrepasando los límites de su dosificación y concentración, lo que trae consigo efectos perjudiciales y daños al organismo como 


\section{Tratamiento de intoxicación aguda por acetaminofén}

Vol. 3, núm. 2., (2019)

Norma Betsabe Arellano Hernández; Karla Estefanía Burgos Cedeño; Karen Mabel Sornoza Moreira; Sandra Lissette Arregui Romero

consecuencia de la intoxicación medicamentosa. La intoxicación aguda con acetaminofén está asociada a riesgos hepáticos, coagulopatía, riesgos renales, compromiso neurológico y hasta la muerte.

Asimismo, si las personas que adquieren este medicamento sin prescripción médica o, aún con prescripción, no siguen las instrucciones de la misma o las contenidas en la etiqueta del empaque corre el riesgo de tomar demasiado acetaminofén accidentalmente. Igualmente, puede correr este riesgo quien tome más de un producto que contenga acetaminofén. "Tomar demasiado acetaminofén puede causar daño hepático, algunas veces tan grave que requiera un trasplante de hígado u ocasione la muerte". (Biblioteca Nacional de Medicina de los Estados Unidos, 2017)

En virtud de lo cual, la presente investigación revisa y plasma el tratamiento de la intoxicación aguda causada por acetaminofén en pacientes de cualquier grupo etiológico, con énfasis en la población infantil que es la más susceptible y donde se presentan los mayores índices de este tipo de problema. Asimismo, trata de puntualizar aspectos generales del antídoto que se usa para el tratamiento de este tipo de intoxicación tan importante y desconocida en la población mundial.

\section{Materiales y Métodos.}

Para el desarrollo de la presente investigación se usaron computadores personales con conexión a internet, con el propósito de exponer acerca del tratamiento de intoxicación aguda con acetaminofén, en razón de lo cual, se enmarca en un diseño de investigación documental o bibliográfica. 


\section{Tratamiento de intoxicación aguda por acetaminofén}

Vol. 3, núm. 2., (2019)

Norma Betsabe Arellano Hernández; Karla Estefanía Burgos Cedeño; Karen Mabel Sornoza Moreira; Sandra Lissette Arregui Romero

Esta investigación se limita a la búsqueda y revisión sistemática de material bibliográfico que, por una parte, es accesible mediante el uso de diversas bases de datos, entre las que figuraron: MedlinePlus, PubMed, Biblioteca Virtual de la Salud (BVS), SciELO, Dialnet y ELSEVIER, entre otras; mediante las cuales se sintetizó la mejor evidencia disponible.

Se realizó una búsqueda aleatoria y consecutiva, usando las expresiones "tratamiento de intoxicación aguda", "tratamiento de intoxicación aguda con acetaminofén", "tratamiento pediátrico de intoxicación con acetaminofen", "acetaminofén” y "acetilcisteína", lo que aproximadamente resultó en más de un centenar de miles de registros bibliográficos que luego se fueron filtrando bajo los criterios de idioma español, relevancia, correlación temática y fecha de publicación en los últimos cinco años, sin descartar por tipo de material bibliográfico.

Por último, se le da la correspondiente lectura crítica y análisis de toda esa evidencia científica, lo que resultó consecutiva y consensuadamente en el contenido del presente trabajo.

\section{Resultados.}

\section{Intoxicación Aguda por Acetaminofén en Pacientes Pediátricos}

Una de las poblaciones más susceptibles ante las intoxicaciones, especialmente con acetaminofén, es la población infantil. El acetaminofén es uno de los antitérmicos y analgésicos más usados en los niños. La venta libre de este medicamento lo hace accesible a los padres, representantes o cuidadores de infantes y, por tanto, su administración muchas veces es llevada a cabo sin supervisión médica. Frecuentemente los padres incurren en errores de dosificación del medicamento, ajustan dosis desconociendo la relación que existe entre el peso del niño, la 


\section{Tratamiento de intoxicación aguda por acetaminofén}

Vol. 3, núm. 2., (2019)

Norma Betsabe Arellano Hernández; Karla Estefanía Burgos Cedeño; Karen Mabel Sornoza Moreira; Sandra Lissette Arregui Romero

concentración del medicamento, la cantidad a administrar y la frecuencia de la toma y el efecto deseado, lo que conlleva a una sobredosis y predispone al niño a una intoxicación involuntaria.

Mintegi Raso (2012) al respecto refiere que los fármacos, a nivel mundial, son el tipo de tóxico con más frecuencia asociado en las intoxicaciones pediátricas, entre ellos los antitérmicos como el paracetamol. En España la ingesta accidental de una sobredosis de paracetamol constituye el 15\% del total de este tipo de intoxicaciones. Asimismo, en los últimos años se ha presentado un incremento de las intoxicaciones secundarias ocasionadas por errores de dosificación del paracetamol líquido, posiblemente ocasionadas por cambios en la jeringa dosificadora. (p. 4,6)

Manejo.

Triviño, Martínez, \& Luaces (2012) recomiendan en principio una valoración inicial y estabilización, aunque el paciente se presente asintomático, ya que el diagnóstico temprano constituye las bases del éxito del tratamiento, posteriormente se debe evaluar el riesgo para la valoración inicial del riesgo que presenta el paciente, es necesario plantearse preguntas para precisar un diagnóstico y su correspondiente terapia. Aquellos pacientes que hayan ingerido dosis no tóxica de paracetamol, es decir, dosis menores a $200 \mathrm{mg} / \mathrm{kg}$ o menores a $8 \mathrm{~g}$ en niños mayores de 6 meses, que carecen de factores de riesgo, podrán ser remitidos a sus hogares indicando a sus padres mantener al paciente observado. En tercer lugar, se debe proceder a descontaminar las vías digestivas aplicables a casos de ingestas entre las últimas una a dos horas, para la cual se administra carbón activado a $1 \mathrm{~g} / \mathrm{kg}$. Por último, se realizan exploraciones complementarias que por medio de la analítica permitirán diagnosticar el grado de intoxicación y prescribir el tratamiento más eficaz. (122-125) 


\section{Tratamiento de intoxicación aguda por acetaminofén}

Vol. 3, núm. 2., (2019)

Norma Betsabe Arellano Hernández; Karla Estefanía Burgos Cedeño; Karen Mabel Sornoza Moreira; Sandra Lissette Arregui Romero

\section{Tratamiento.}

"La piedra angular en el tratamiento de la intoxicación por paracetamol es su antídoto específico, la N-acetilcisteína (NAC)”, el cual es un precursor metabólico del glutatión, actúa en el relleno de sus depósitos hepáticos y se conjuga con la NAPQ1 (derivado metabólico que destruye los hepatocitos), ocasionando la anulación de su efecto hepatotóxico. Su máxima eficacia se consigue cuando se administra dentro de las primeras ocho horas posteriores a la ingesta del fármaco, por tal razón es fundamental. en estos casos, actuar con la mayor rapidez posible. Este tratamiento no se encuentra libre de presentar complicaciones, por lo tanto, es imperioso que se inicie solo en los casos de comprobado riesgo hepático. (Triviño, Martínez, \& Luaces, 2012, p. 126)

Intoxicación Aguda por Acetaminofén en Pacientes Adultos

Oliva \& Canals (2018) proponen para el manejo general de las intoxicaciones agudas un cuadro sencillo que contiene: 1. Medidas de soporte general (ABCD), 2. Medidas para disminuir la absorción del tóxico (inducción del vómito, lavado gástrico, carbón activado, lavado intestinal, entre los principales), 3. Eliminación del tóxico y 4. Antídotos. (p. 52)

Un diagnóstico temprano resulta fundamental para el tratamiento de la intoxicación aguda con acetaminofén por cuanto estas intervenciones tempranas reducen el riesgo de daño hepático significativamente. El factor tiempo es determinante además en la selección del tipo de tratamiento, ya que este depende de las horas transcurridas desde la ingesta del medicamento. Las primeras veinticuatro horas de intoxicación el paciente puede presentar una clínica inespecífica, 


\section{Tratamiento de intoxicación aguda por acetaminofén}

Vol. 3, núm. 2., (2019)

Norma Betsabe Arellano Hernández; Karla Estefanía Burgos Cedeño; Karen Mabel Sornoza Moreira; Sandra Lissette Arregui Romero

con dolor abdominal, fatiga, fiebre, anorexia, entre otros. Posterior a este lapso el paciente puede evolucionar hasta una encefalopatía durante los primeros siete días. (Rojas, Quesada, \& Esquivel, 2019)

El carbón activado es una buena opción para disminuir, previo al tratamiento, la absorción del tóxico.

Vargas Castro (2016) recomiendan para "pacientes que se presentan rápidamente posterior a la ingestión (antes de 4 horas) se recomienda la descontaminación gastrointestinal utilizando carbón activado a $1 \mathrm{~g} / \mathrm{kg}$ (máximo 50g) ya que limita la absorción del fármaco". (p. 3)

El antídoto básico de la intoxicación aguda con acetaminofén es la acetilcisteína. Giménez, Garay, \& Anta (2016) en la Guía de Antídotos en Hospital Universitario Marqués de Valdecilla, España, se refieren la posología de este medicamento en adultos y adolescentes, esto es, persona que supere los $40 \mathrm{~kg}$, en cuanto a que la dosis debe ser calculada usando el peso real del paciente. Acotan que en aquellos casos de pacientes que presentan obesidad se debe considerar aplicar un umbral máximo para el peso de $110 \mathrm{~kg}$. Mencionan además que el ciclo completo de tratamiento está conformado por tres perfusiones intravenosas consecutivas. "La acetilcisteína debe diluirse antes de administrar (no IV directo), preferentemente utilizar glucosa al 5\%, aunque es posible utilizar cloruro de sodio al $0,9 \%$ ". Se deben administrar los preparados de forma secuencial y sin interrupciones entre cada perfusión. (p. 11,12)

La N-acetilcisteína es básica en la prevención de la hepatoxicidad, restaura las reservas hepáticas de glutatión. "La clave para la efectividad del tratamiento es iniciarlo en las primeras 8 


\section{Tratamiento de intoxicación aguda por acetaminofén}

Vol. 3, núm. 2., (2019)

Norma Betsabe Arellano Hernández; Karla Estefanía Burgos Cedeño; Karen Mabel Sornoza Moreira; Sandra Lissette Arregui Romero

horas de la ingestión aguda". En la actualidad, se utilizan dos tipos de protocolos: en principio, vía intravenosa, de 20 horas y vía oral, de 70 horas. Para pacientes que se presentan con: emesis, contraindicaciones para la administración oral y/o fallo hepático, les favorece la vía intravenosa. En base a muchos autores, la duración de estos tratamientos debe ser individualizada. Es recomendable revalorar las enzimas hepáticas y concentración sérica del acetaminofén 18 horas después de haber iniciado el antídoto. En caso de permanecer alterados, es recomendable continuar el tratamiento e iniciar una nueva valoración a las 12 horas. El momento de suspender el tratamiento es cuando el ALT este reduciéndose, la concentración sérica del medicamento debe ser indetectable y el INR debe ser menor a dos. (Vargas Castro, 2016, p. 4,5)

Prescott et al. citados por Corominas \& Nogué (2019), explican que el régimen tradicional para la administración endovenosa de la acetilcisteína se lleva a cabo en tres fases: la primera dosis de carga de $150 \mathrm{mg} / \mathrm{kg}$ en 15 minutos, la segunda de $50 \mathrm{mg} / \mathrm{kg}$ durante 4 horas y la tercera de 100 $\mathrm{mg} / \mathrm{kg}$ durante 16 horas. Esto equivale al protocolo de $300 \mathrm{mg} / \mathrm{kg}$ en 20 horas. Este régimen tradicional se ha mantenido invariable hasta la actualidad con la excepción de una pequeña modificación en el tiempo de infusión de la dosis de carga, que pasó de 15 minutos a una hora a los fines de minimizar los efectos adversos. (p. 2)

Para Park, Dear, \& Antoine (2014) "los efectos adversos de la acetilcisteína incluyen erupción cutánea, urticaria, vómitos y reacciones anafilactoides, que pueden (aunque rara vez) sea fatal. Estos pueden reducirse mediante el uso de nuevos regímenes para el tratamiento con acetilcisteína”. (p. 4) 


\section{Tratamiento de intoxicación aguda por acetaminofén}

Vol. 3, núm. 2., (2019)

Norma Betsabe Arellano Hernández; Karla Estefanía Burgos Cedeño; Karen Mabel Sornoza Moreira; Sandra Lissette Arregui Romero

Por otra parte, con relación a los efectos adversos de la acetilcisteína, Mancipe, Fernández, \& Fernández (2010) reportan en su trabajo, que la administración endovenosa de este antídoto ha ocasionado hipotensión, urticaria y reacciones anafilactoides leves y transitorias. La relación de estos efectos adversos se asocia directamente con la dosis y rata de administración. Asimismo, por vía oral, estos efectos adversos se han presentado a nivel gastrointestinal tales como náuseas y vómito. (p. 225, 226)

Corominas \& Nogué (2019) refieren que existe un nuevo régimen para la administración de la acetilcisteína que busca optimizar su uso disminuyendo los efectos adversos, se trata del esquema SNAP (las siglas del inglés Scottish and Newcastle Antiemetic Pretreatment Paracetamol Poisoning Study Regim). Este régimen consiste en combinar las dos primeras infusiones de las tres estipuladas para el régimen estándar, las cuales son "administradas con una velocidad inicial más lenta, esto es, $200 \mathrm{mg} / \mathrm{kg}$ durante 4 horas (dosis de carga), seguida de una infusión final de $100 \mathrm{mg} / \mathrm{kg}$ durante 16 horas (300 mg/kg en 20 horas)". Con base al nuevo régimen, los estudios han demostrado una incidencia menor y significativa de reacciones anafilactoides. (p. 3)

\section{Conclusiones}

El tratamiento básico de la intoxicación aguda con Acetaminofén está constituido por la Acetilcisteína, antídoto que actúa en la prevención del daño hepático, en razón de lo cual, resulta vital el diagnóstico temprano y la consecutiva administración de este fármaco. 


\section{Tratamiento de intoxicación aguda por acetaminofén}

Vol. 3, núm. 2., (2019)

Norma Betsabe Arellano Hernández; Karla Estefanía Burgos Cedeño; Karen Mabel Sornoza Moreira; Sandra Lissette Arregui Romero

La población infantil resulta la más susceptible a intoxicación aguda con acetaminofén involuntariamente, no obstante, es una de las principales causas tanto de intoxicación voluntaria como involuntaria en adultos.

En el manejo de estas intoxicaciones deben complementarse una buena valoración clínica (de ella depende la premura de la administración del tratamiento y, por ende, evitar lo más posible daños en el organismo), la estabilización del paciente y la prevención de la absorción del tóxico son de gran importancia para la administración del tratamiento y que este logre los mejores resultados, la eliminación del agente tóxico con el menor daño posible al organismo del paciente. Por último, la terapia de soporte va a coadyuvar en la recuperación total del paciente.

En cuanto al pronóstico del paciente con intoxicación aguda con acetaminofén, el diagnóstico y el tratamiento temprano va a modificarlo favorablemente en la mayoría de estos casos. La morbilidad en estos casos está íntimamente relacionada con el lapso de tiempo que transcurre entre la ingesta de la sobredosis de acetaminofén y la administración del antídoto (Nacetilcisteína).

Por último, no existe unificación de criterios a nivel internacional acerca del régimen para la administración del tratamiento con este antídoto, no obstante, muchos países e instituciones se han adherido durante años al método tradicional con algunas variaciones realizadas en base a la evidencia de nuevas investigaciones. Desde el 2015 y 2016, estudios han permitido desarrollar regímenes más modernos que ayudan a tratar la intoxicación aguda con acetaminofén evitando en mayor medida sus efectos tóxicos. 


\section{Tratamiento de intoxicación aguda por acetaminofén}

Vol. 3, núm. 2., (2019)

Norma Betsabe Arellano Hernández; Karla Estefanía Burgos Cedeño; Karen Mabel Sornoza Moreira; Sandra Lissette Arregui Romero

\section{Referencias Bibliográficas}

Biblioteca Nacional de Medicina de los Estados Unidos. (14 de abril de 2017). MedlinePlus. Recuperado el 05 de agosto de 2019, de https://medlineplus.gov/spanish/druginfo/meds/a681004-es.html

Corominas, N., \& Nogué, S. (enero - mayo de 2019). Nuevas pautas de dosificación de la Nacetilcisteína en la intoxicación por paracetamol. Boletín de Antídotos de Cataluña, 2(1), 1-15. Recuperado el 03 de agosto de 2019, de http://www.fetoc.es/novedades/BAC_n1_2019.pdf

De la Torre, M. (2014). Intoxicaciones más frecuentes. Pediatría Integral, 18(5), 280-290. Recuperado el 03 de agosto de 2019, de https://www.pediatriaintegral.es/wpcontent/uploads/2014/xviii05/01/280-290.pdf

Giménez, T., Garay, C., \& Anta, J. (2016). GUIA DE ANTÍDOTOS EN HUMV. Hospital Universitario Marqués de Valdecilla, Centro de Información de Medicamentos. Santander: Servicio de Farmacia del Hospital Universitario Marqués de Valdecilla. Recuperado el 04 de agosto de 2019, de http://www.humv.es/estatico/docs2016/guia_de_antidotos_en_humv.pdf

Mancipe, L., Fernández, D. C., \& Fernández, D. G. (01 de diciembre de 2010). INTOXICACIÓN POR ACETAMINOFÉN. Revista Med, 18(2), 221-227. Recuperado el 04 de agosto de 2019, de http://www.scielo.org.co/pdf/med/v18n2/v18n2a08.pdf

Mayo Clinic. (24 de enero de 2019). mayoclinic.org. Recuperado el 05 de agosto de 2019, de https://www.mayoclinic.org/es-es/first-aid/first-aid-poisoning/basics/art-20056657

Mintegi Raso, S. (2012). Introducción. En G. d. Pediatría, Manual de Intoxicaciones en Pediatría (Tercera ed., pág. 476). Madrid, Majadahonda, España: Centro Español de Derechos Reprográficos. Recuperado el 01 de agosto de 2019, de https://seup.org/pdf_public/gt/intox_manual3_enr.pdf

Oliva, J. R., \& Canals, M. (2018). Sociedad Española de Medicina de Familia y Comunitaria. Recuperado el 08 de agosto de 2019, de https://www.semfyc.es/wpcontent/uploads/2018/05/IntoxicacionesAgudas.pdf

Organización Mundial de la Salud. (2017). who.int. Recuperado el 02 de agosto de 2019, de https://www.who.int/ipcs/poisons/es/

Park, B. K., Dear, J. W., \& Antoine, D. J. (2014). Paracetamol (acetaminophen) poisoning. Clinical Evidence, 10(2101), 1-15. Recuperado el 03 de agosto de 2019, de https://www.ncbi.nlm.nih.gov/pmc/articles/PMC4610347/pdf/2015-2101.pdf 


\section{Tratamiento de intoxicación aguda por acetaminofén}

Vol. 3, núm. 2., (2019)

Norma Betsabe Arellano Hernández; Karla Estefanía Burgos Cedeño; Karen Mabel Sornoza Moreira; Sandra Lissette Arregui Romero

Rojas, C., Quesada, M., \& Esquivel, N. (2019). Intoxicación por acetaminofén: diagnósticoy abordaje en el adulto. Revista Médica Sinérgia, 4(7), 1-14. Recuperado el 03 de agosto de 2019, de https://revistamedicasinergia.com/index.php/rms/article/view/257/593

Triviño, M., Martínez, L., \& Luaces, C. (2012). Intoxicaciones medicamentosas más habituales. En G. d. Pediatría, Manual de Intoxicaciones en Pediatría (pág. 476). Madrid, Majadahonda, España: Centro Español de Derechos Reprográficos. Recuperado el 02 de agosto de 2019, de https://seup.org/pdf_public/gt/intox_manual3_enr.pdf

Vargas Castro, M. P. (2016). Intoxicación por Acetaminofén en Adultos. Medicina Legal de Costa Rica - Edición Virtual, 33(1), 1-7. Recuperado el 03 de agosto de 2019, de https://www.scielo.sa.cr/pdf/mlcr/v33n1/1409-0015-mlcr-33-01-00103.pdf

$$
\text { (2) }(1)(9)
$$

RECONOCIMIENTO-NOCOMERCIAL-COMPARTIRIGUAL

CC BY-NC-SA

ESTA LICENCIA PERMITE A OTROS ENTREMEZCLAR, AJUSTAR Y CONSTRUIR A PARTIR DE SU OBRA CON FINES NO COMERCIALES, SIEMPRE Y CUANDO LE RECONOZCAN LA AUTORÍA Y SUS NUEVAS CREACIONES ESTÉN BAJO UNA LICENCIA CON LOS MISMOS TÉRMINOS. 